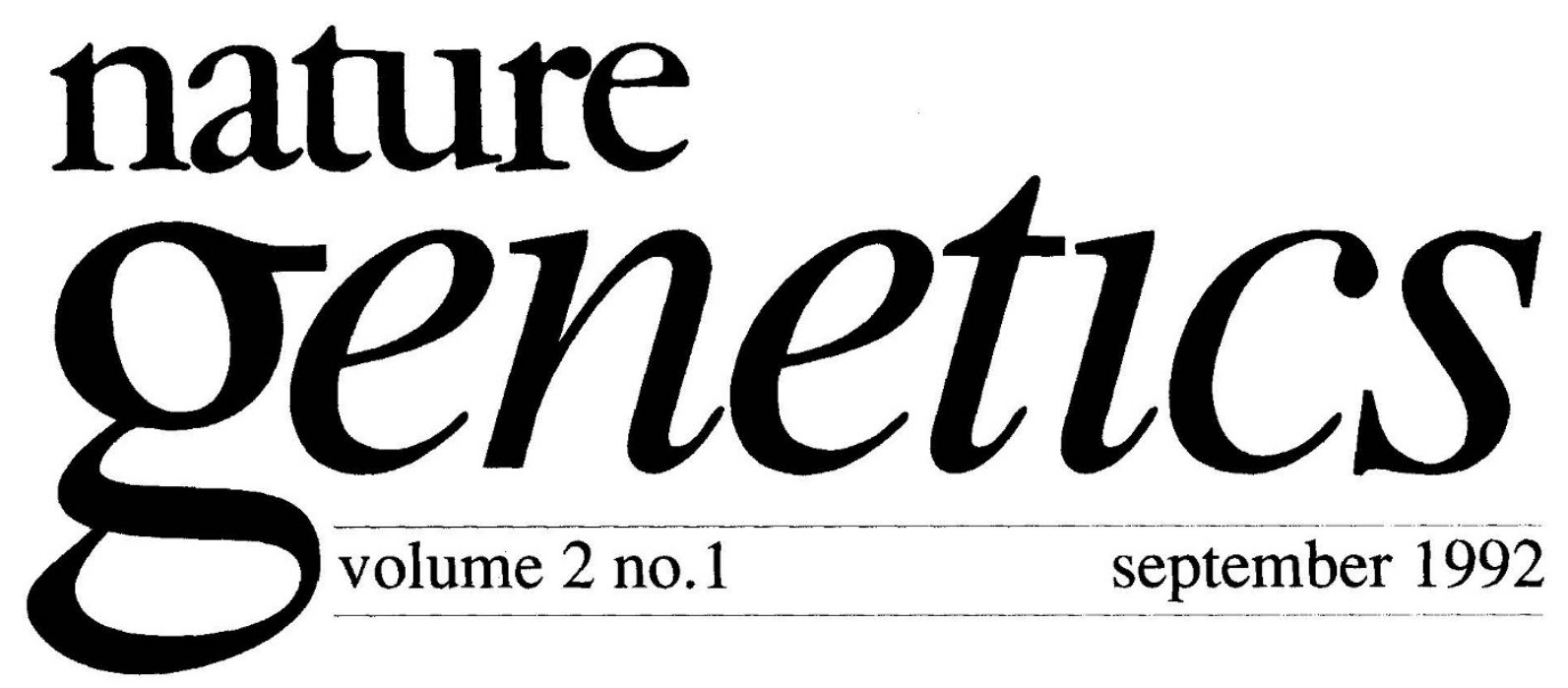

\title{
Signs of an imprinted domain
}

The field of genetic imprinting, that so galvanized biologists last year with the identification of three murine genes whose alleles are differentially expressed depending on their parent of origin ${ }^{1-3}$ (parental imprinting in other words) is stirring again after a relatively quiet few months. The existence of imprinted genes was not totally unexpected - it was well known that a combination of maternal and paternal genes is required for normal mouse embryological development. On the other hand, the absence of a parental contribution for certain chromosomes does not produce any discernible phenotypic effect, so the total number of imprinted genes in the genome may be small.

The three mouse genes that were shown last year to be imprinted include the ligand ${ }^{1}$ and one of the receptors ${ }^{2}$ for insulin-like growth factor II ( $I g f-2$ and $I g f 2 r$ ), which map to mouse chromosomes 7 and 17 respectively. Igf $2 r$ was one of four genes identified in an approximately one megabase segment embracing the $T$-associated maternaleffect (Tme) mutation, which is lethal if deleted from the maternal - but not the paternal chromosome (an example of paternal imprinting, therefore, in which the Tme allele on the paternally derived chromosome is expressed poorly or not at all). Barlow ${ }^{2}$ and her colleagues found that Igf $2 r$ is not expressed from the paternal allele, whereas three defined genes nearby are expressed from both chromosomes, making Igf $2 r$ a tempting candidate for the Tme locus - at least until a recent report presented genetic evidence that the two loci may be distinct ${ }^{4}$.
The third known imprinted gene ${ }^{3}$, called H19, maps close to Igf-2 at the distal end of mouse chromosome 7. Despite their proximity (evident from linkage analysis) they are imprinted in opposite directions: that is, Igf-2 is active only from the paternal gene, whereas H19 is expressed exclusively from the maternal allele. The function of H19 is not known - Shirley Tilghman's group at Princeton University has shown that overexpression of $\mathrm{H} 19$ in transgenic mice results in embryonic lethality, and yet comparison of the $H 19$ gene sequence from several mammalian species suggests that the gene does not encode a protein and may therefore act at the RNA level (reviewed in ref. 5). It may not be coincidence that $X i s t$, the only gene known to be expressed exclusively from the inactive $\mathrm{X}$ chromosome and which is thought to play a central role in the process of $\mathrm{X}$ inactivation, similarly does not contain an open reading frame ${ }^{5}$.

Of course, it is also rather improbable that the opposite imprinting of $I g f-2$ and $I g f 2 r$ is mere coincidence, and it has been argued that such effects have arisen due to natural selection - the maternally expressed Igf- 2 receptor acting as a 'sink' to control the level of Igf-2, an embryonic growth factor, which might have deleterious consequences for the population ${ }^{6}$. But with little firm information on the molecular mechanism of imprinting or its likely physiological role (maintenance of species diversity has also been proposed), a sensible strategy is to acquire more information about the structure and organization of the imprinted genes. To this end, the paper 
from Tilghman and colleagues on page 61 of this issue $^{7}$ clarifies the physical relationship between Igf2 and H19. It turns out that these two murine genes are oriented in the same direction and separated by only 90 kilobases $(\mathrm{kb})$. Eighteen kb upstream of Igf2 is the insulin-2 locus, which is believed not to be imprinted. The authors propose that common transcriptional elements for the pair of imprinted genes may play a part in determining their specific allelic expression, and that this segment of chromosome 7 may constitute an imprinted 'domain'. Certainly the twogenes share avery similar pattern of temporal and tissue-specific expression. Further scrutiny of this region may reveal important clues about the imprinting process and the domain hypothesis, such as the question of whether other (imprinted?) genes may actually exist between $I g f-2$ and $H 19$.

These genes are of interest for human geneticists as well. The Ins-2/Igf-2/H19 synteny is conserved on human chromosome $11 \mathrm{p} 15.5$ - indeed, Tilghman's group also finds that, in humans, IGF2 and H19 are physically linked, and are a maximum of $200 \mathrm{~kb}$ apart $\mathrm{t}^{5}$. Interestingly, this is the region in which paternal isodisomy (two copies of the father's loci) has been associated with sporadic cases of Beckwith-Wiedemann syndrome (BWS) ${ }^{7}$, which is characterized by gigantism and predisposition to childhood tumours. A causative role for IGF2 in BWS is supported by the overexpression of IGF2 in tumours from BWS patients, but more interestingly from the observation of the larger than normal size of chimaeric mice containing paternal duplications of the distal end of chromosome 7 , suggesting a dosage effect of the paternally expressed Igf-2 (ref.8). However, H19, the first mouse gene to have been shown to be monoallelicly expressed in humans ${ }^{9}$, could also be involved in some aspects of BWS, perhaps because of the loss of a tumour suppressor function ${ }^{5,8,10}$. The speculation concerning these remarkable genes should lead shortly to substance.

1. DeChiara, T.M., Robertson, E.J. \& Efstratiadis, A. Cell 64, 849-859 (1991).

2. Barlow, D.P. et al. Nature $349,84-87$ (1991).

3. Bartolomei, M.S. et al. Nature 351, 153-155 (1991).

4. Forejt, J. \& Gregorova, S. Cell 70, 443-450 (1992).

5. Bartolemi, M.S. \& Tilghman, S.M. Sem. devl. Biol. 3, 107117 (1992)

6. Haig, D. \& Graham, C. Cell 64, 1045-1046 (1991).

7. Zemel, S. et al. Nature Genet. 2, 61-65 (1992).

8. Junien, C. Curr. Opin. genet. Devl. 2, 431-438 (1992).

9. Zhang, Y. \& Tycko, B. Nature Genet. 1, $40-44$ (1992).

10. Little, M., van Heyningen, V \& Hastie, N. Nature 351, 609610 (1991).

\section{Cystic fibrosis mice}

By a strange quirk of timing, the report by Whitsett et al.on page 13 of this issue, of healthy transgenic mice expressing the human cystic fibrosis transmembrane regulator (CFTR) may be contrasted with the long-awaited production of homozygous CFTR-deficient mice, which has just been announced by Beverly Koller and coworkers at the University of North Carolina (Science 257, 1083-1088; 1992). The Science report marks the first successful attempt among several groups worldwide to 'knock out' the CFTR gene in mice by homologous recombination, thus producing the first authentic animal model for cystic fibrosis $(\mathrm{CF})$. For those working on the mechanics of gene targeting, there seems to be no magic solution to the difficulties in disrupting the CF gene that have beset researchers for the past two years. Targeting efficiency often varies between experiments, but finally one experiment yielded embryonic stem cells in which the disrupted CFTR gene (containing a premature stop codon) had integrated correctly and could then be bred into the mouse germline.

The mice that are homozygous for the defective CF gene reveal many important similarities but also some curious differences from human patients. In almost all cases, the affected mice are smaller than normal and die before they are 40 days old due to peritonitis caused by severe intestinal blockage (they would normally be expected to live for two years or so). This obstruction is reminiscent of meconium ileus, an early sign of CF in newborns. However, other organs including the pancreas, liver and reproductive tracts appeared relatively normal, and there is evidence that some male mice are fertile. Most important, although the upper respiratory tract showed some pathological changes, there were no signs of bacterial infection in the lungs - the most critical component of human CF. If the intestinal problems can be overcome, thus prolonging the mouse lifespan, susceptibility to bacterial infection may become apparent and accessible to study. It should also be possible tocombine the transgenicand genetargeting technologies to witness the effect of reintroducing and expressing CFTR in a variety of cell types which otherwise lack the protein. The CFTRdeficient mice are being made available through the Jackson laboratories and will be a boon to $\mathrm{CF}$ research.

nature genetics volume 2 september 1992 